\title{
Sexual Health Challenges amongst Low-Females Profile in Urban Area: The Case of Maroua-Cameroon
}

\author{
Dr. Lucy FONJONG UDIKOH * \\ Senior Lecturer, Faculty of Arts, Letters and Social Sciences Department Anthropology University of Yaounde-Cameroon \\ Article History \\ Received: 21.08.2020 \\ Accepted: 07.09.2020 \\ Published: 30.09.2020 \\ Journal homepage: \\ https://www.easpublisher.com/easjhcs

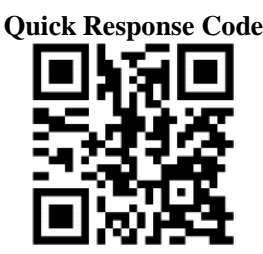 \\ Abstract: This is an ethnographic study about knowledge proposal and effective usage of \\ contraception among young females in the extreme north region of Cameroon as there is a \\ global differentiation in family planning in terms of practice and typology of methods used. \\ Looking at women's unmet needs, some constraints linked to low profile like distance, \\ knowledge, cost of contraceptives, availability, biases, as well as cultural notions to \\ unmarried youth plays a very vital role. To mention: cultural and religious disapprovals to \\ contraception, protests from a partner, in-law or family members, scarce knowledge, dread \\ and obstructive gender norms restraining procreative agency are evident. Using qualitative \\ research method, participatory purpose sampling, focus groups, interviews as well as \\ freelisting, ministered in our data collection, life stories and direct observation besides \\ documentaries and internet resources were explored to have this write-up. Our objective is: \\ To unveil women's suffering status and the implications of low socio-cultural profile to \\ health and sexual activities amongst members. This study is vital to hail policymakers to \\ handle matters of marriage and reproductive health in Cameroon and the Extreme North \\ region in particular. Speaking concretely about unmet need for family planning entails \\ employing interferences to wrestle limitations on both the individuals and identifying means \\ in which sources and request matters touch every party. \\ Keywords: Sexuality, Health, Low, Female, Profile.
}

Copyright (C) 2020 The Author(s): This is an open-access article distributed under the terms of the Creative Commons Attribution 4.0 International License (CC BY-NC 4.0) which permits unrestricted use, distribution, and reproduction in any medium for non-commercial use provided the original author and source are credited.

\section{INTRODUCTION}

Although words can reveal cultural beliefs, action speaks better and the term "sex in our case." has in the past, referred both to sexuality and to someone's biologic sex: male or female. Currently, the word sex still refers to sexuality, with "gender" progressively termed the sets male, female, as well as other gender possibilities. Why?

This is because societies that are vastly separated by gender, gender dealings at times could be view unfriendly or oppositional with one of the genders (usually female). Meanwhile in some cases, she is seen as possibly frightening. Cases of the natural phenomena in women like bodily fluids, such as menstrual blood and vaginal secretions, can be perilous, undesirable to men, "impure," and "contaminating," particularly in ritual contexts while in some circumstances, menstrual blood is associated with positive power and be illustrious openly with elaborate community rituals, as among the Bemba in southern Africa. Hence! subsequent monthly flows bring special privileges. Men in some small-scale societies go through ritualized nose-bleeding, sometimes called "male menstruation," though the meanings are quite complex. With such mindset sexuality to anthropologists is seen to be diverse and understood differently in context where the status quo may arise.

In the meantime, this study is focus on the sexual view and practices carried out in Maroua urban centre of the far North of Cameroon with woman as target.

\section{Case Study}

The Extreme North region is a homogeneity area compressed of many ethnics and religions but is Islam quite visible. It is a major religion in this area. These groups include: the Peuls, Giziga, Mofou, Moundang,Toupouri, Massa, Gidar, and others. It has six Divisions and a number of sub divisions and according to RGPH 1987, the populations of Maroua its main capital was more than 123,000 and has been on an increased. This can be reconstructed to the different dynamics in the socio-cultural infrastructures like the creation of the University besides many primaries, secondary and professional institutions located in the region. The population is highly involved with commerce, animal rearing and farming of millet, maize, cotton, groundnuts and so on. The main food cultivated and consumed here is millet. It is purely a Sahel area with few rivers like the Logone. Touristic activities are 
very identical in this area with outstanding features of the Kapsiki and Rhumsiki areas. Being an artistic region, one could enjoy many designs of dresses, shoes, bags and are made out of animal skin. Though it is an emerging town with many government projects in view, it has a regional hospital in the capital town of Maroua to serve it increasing population. Many homes still go without food and high birth rates are experienced. Many seek informal health treatment and clandestine methods of services are prevalence.

\section{Methodology}

This study highly applied participatory observations, and interviews, documentaries were also examined with local literature equally explore. One of such is the study carried out by The Association to Combat Violence against Women-Extreme North (ALVF-EN). This is a feminist organization that aims to eliminate all forms of violence affecting women and girls, including early and forced marriage, in the northern regions of Cameroon. ALVF-EN is a sponsored study to better understand the practices and help its members and other organizations to effectively advocate against early child marriage as well as promote women's empowerment. Specifically, ALVFEN plans to use the findings to advocate for the implementation of a new Code of the Family and Person.

By this research, the aim and methodology was to determine the nature and scale of children and early forced marriage in Cameroon. The Institut Supérieur $d u$ Sahel at the University of Maroua (Cameroon), in partnership with ALVF-EN, surveyed individuals and families on their views of early and forced marriage and its main drivers. They also examined the legal context in which these marriages take place and the profile of risking most girls and women. Focus groups, in-depth interviews and surveys were used to collect data on perceptions in all ten regions of Cameroon, urban and rural areas alike.

\section{Research Design}

Our main method used in collecting data was direct observation and participation so as to maintain our objectivity. Through this technique we observed the lifestyles, attitudes and health seeking sources especially when somebody is suffering from a venereal disease. We equally gathered data through informal interview like in bus and trains stations. Radio and television programs and news on the locality was one of our major sources of information. Thus data was collected during interviews, 03 focus group discussions and observations, over a period of 2 years. Formal discussions with people from other regions were carried out to avoid ethnocentrism. Though our study was basically primary since participant observation was highly involved, medical personnel were equally questioned to acquire knowledge of social behavior and clinical based information on matters of reproductive health, family planning and sexuality. In addition, many people think traditional practitioners, are highly contacted during illness than hospitals for health information. We gave attention to them so as to understand this perception.

\section{Objectives}

To unveil women's suffering status and the implications of low socio-cultural profile to health and sexual activities.

Documentation was explored for secondary data to have an idea of what authors have written in relation to the topic. Students also participated to bring in some useful information concerning the persistence of diseases due to the non-collaborative attitude of the population to stay away from their daily life. Practically, we worked with 120 informants though we targeted 250, many of whom were women. In all, we had 90 female informants and 30 male informants. We worked with 01 traditional healer, 01 medical doctor and 02 nurses and some teachers. This sample was to permit us to reach our objective. Their different genetic, professional and social backgrounds coupled with what we observed helped to broaden our knowledge and originality of this work. Strategies for the Future Empowerment through education are ideally seen as a continuous holistic process with cognitive, psychological, economic and political dimensions in order to achieve emancipation. The importance of doing participatory and action research was emphasized.

\section{Health and information}

Ambiguity is one of the occurrences of our times but more impacting, in describing the far north region for a keen listener to MOKOLO, Cameroon where conditions are often traumatic for women and girls in Cameroon's Far North Region. Rape, violence, child marriage, rites, maternal death, etc are too accustomed involvements in this part of the country and the girl child is at center of this scenario. Nonetheless, these severe apprehensions are carried out in head-on unforeseen place: The local 95.8 FM radio station is a radio frequency that routinely bid women and girls a hint into another way of living as from one o'clock in the afternoon. - By this program, a world where female are endowed valued, and their voices are heard is put in place. Through this, one could see worries to women's health and rights. Meanwhile,

UNFPA's definition of Violence involving men and women, in which the female is usually the survivor; is derived from unequal power relationships between men and women. Violence here is directed specifically against a woman because she is a woman, or affects women disproportionately. It includes, but is not limited to, physical, sexual and psychological harm (including intimidation, suffering, coercion, and 
deprivation of liberty within the family, or within the general community). It includes violence which is perpetrated or condoned by the State". (UNFPA Gender Theme Group, 1998) (UNFPA, 1998)

UN Declaration on the Elimination of Violence against Women in 1993 defines VAW as: "Any act of gender-based violence that results in, or is likely to result in, physical, sexual or psychological harm or suffering to women, including threats of such acts, coercion or arbitrary deprivations of liberty, whether occurring in public or private life." (UN Declaration on Violence Against Women, Article 1.)

Proved to this health threat, is that the Far North Region according to 2005 census data, has the second highest child marriage rate in the country among girls aged 12 to 14 . Besides, debates about women's reproductive health and rights are deliberated taboo, leaving women and girls deprived of access to critical information and care. All of this contributes to Cameroon's amazing maternal death rate, one of the highest in the world. One programme, in particular, offers a platform for women to express opinions rarely heard in public. In 'Débat au féminin' - Debates of Women - women discuss issues widely considered forbidden, including sexual violence, sexually transmitted infections such as HIV, family planning, and child marriage.

\section{Connecting culture and illness}

Anthropologists use culture to define settings in any community. To that effect, perception based that culture in its form build the human spirit. Thus culture could be asserted to be all deliberate and conscious activities done by man as a rational being and as a member of the society with other manifestations which comes as a result of his society. Meanwhile illness on its part could be termed as an alternation of health, as well as the modification and unfavourable changes in the steady nature of human being. Meanwhile WHO defines health as a state of complete physical, mental, and social well-being and not merely the absence of disease or infirmity. - Preamble to the Constitution of the World Health Organization, 1946.

By this ideology, culture has a great effect on health and shape people notion on illness through beliefs about the causes of diseases approaches to promoting health, experience and expression of disease and pain. So people's worldview controls the choice of places where patients turn for help and the treatment preferred. Collectivist and individualist cultures can generate different views on human health as well as treatment, diagnosis and causes of diseases. That is the reason why it is always necessary to understand the patient's position in the cultural continuum since information given may be useful to include the extended discussions on the origin of the disease family, diagnosis and treatment. Subsequently, it may be necessary to obtain the consent of the gown up before certain diagnosis and therapy are carried out.

However, sexuality is a vital aspect of human life the more reason why sexual behaviour of young people as well as adult is one of the most significant social and major public-health alarms in current times. As HIV prevalent and exploration on sexual behaviors of people has advanced quickly over the past eras. Connecting acceptance that sexual and reproductive health matters continue to be the leading cause of illhealth among young individual especially at the time when they are sexuality active worldwide. With a growing sexual group in Maroua -Cameroon there is concern. Yet, inquiry on people's sexual health especially among young active female is lacking or little.

Meanwhile, in the light of Kleinman A. (1978:3), an illness is the way a sick person and the members of his or her family or wider societal network perceive, live and respond to symptoms and disability. In such a situation therefore, illness includes a state of bad health, presented by the patient's judgments about how best to cope with the distress and the practical problems in daily life this create. Supporting this phenomenon Jecker N, 1995 et al. stipulate that Health care professionals in primary care settings are often challenged by the cultural differences between themselves and their patients that may inhibit effective and satisfactory health care. It is therefore obvious that knowledge of the factors underlying disease emergence can help focus resources on the key situations and areas worldwide and develop more effective prevention strategies. If we are to protect ourselves against emerging diseases, the essential steps are effective global step. To support the above opinion, it should be noted that three billion people in the world lack most basic element of sanitation--the toilet. The result is an increased exposure of people to disease; diarrhea, the most common disease spread by feces contact, kills two million children a year. Furthermore, $90 \%$ of all infectious disease in developing countries is waterborne.

\section{Sex Education}

In many parts of Cameroon and in the Far North particularly sexuality-related issues have mainly lingered as a taboo. Socio-cultural activities such as religion, economic as well as informal education has made the community to have many strong traditional norms and beliefs relating to sex and sexuality. The Islamic religion is highly practices among members of this area and sex issues are seldom conversed within the family setting. This is quite difficult since it is considered sacred. In the same light like in many parts of the country friendships between girls and boys are still intolerable though not vigorous. The religious 
culture as the case maybe is a place where parents even depress their daughters from meeting or talking with boys. As a result sexual happenings outside a marriage union are not accepted among the majority. Notwithstanding these traditional opinions, a good number of people both young and old are involved in pre- and extra-marital sex.

Although it is broadly believed that people especially the youths have precise sexual health requirements, which differ giving to their age, sex, marital and socioeconomic status engaging on insecure sex could be dangerous. Outcome of sexual vulnerability could be sexually transmitted infections (STIs) and unwanted pregnancies and worst HIV/AIDS. That is the very reason why, many health employees everywhere in the world are struggling to stop it. Or at most decrease risk-taking behaviours of young people. Though, the interest of young people in gaining relevant information and friendly services sometimes are hindered by their profile, the provision of sexual and reproductive health services in far North region is very insufficient. Given that many people have had some notion of modernization without clear understanding of the phenomena. In some cases, there is a common impression that modernization and globalization have caused many young people to delay lengthier afore getting married consequently and this has created more chances for them to devote time in familiar sexual relationships before marriage as they spend more years in schooling and get married well ahead.

In the Nepalese Government for example it is dedicated to providing a package of sexual and reproductive health service to young people. The Government has developed the National Adolescent Health and Development Strategy and the Young People Development Programme. These strategies have visualized adolescent and young people as a key target group for integrated sexual and reproductive health services, with interventions planned to increase knowledge on sexual and reproductive health issues and availability of services. A Country like South Africa as another sample places packet of preventive such as condoms in train stations and other public places to educated its citizen to take measures on protected sex. Such initiatives are quite visible in many other countries. But a region highly given to illiteracy and lack of necessary measures due to their world view could be very challenging as Maroua.

Pokharel S, Kulczycki A, Shakya S. 2006;14:156-61 indicates that Nepalese young people gain information and education on sexual and reproductive health mainly through radio and healtheducation programmes targeted towards the general population. Meanwhile, most sexual and reproductive health services in Nepal are provided through private and public-health centres. These include local pharmacists, public-health practitioners, doctors, nurses, and community health workers. Young people obtain sexual health services when they visit health centres, hospitals, or clinics. However, many such programmes are poorly implemented. Besides Pradhan A., M Strachan - 2003 stipulates that a very few sexual health services, mainly governmental services in rural areas of Nepal, are available. On the contrary, urban areas have more specialized facilities with many sexual health service centres, which young people can access easily. With cultural ideology in the Far North, that sex education is sacred; many young people are limited and challenged to hear about sex issues from friends at school or from self-centered individuals who could use such scenario to exploit them.

One specific program, that offers a stage for women to voice out their sentiments rarely heard in public.is the 'Débat au féminin' - Debates of Women Here, women issues are conversed and broadly wellthought-out forbidden, including sexual violence, sexually transmitted infections such as HIV, family planning, and child marriage.

\section{Challenges in Low profile}

In one hand, culture has great influence on health and colour the perception of health illness through beliefs about the causes of diseases approaches to promoting health, experience and expression of disease and pain. To that effect it controls the choice of places where patients turn for help and the treatment preferred. Collectivist and individualist cultures can generate different views on human health as well as treatment, diagnosis and causes of diseases. That is the reason why it is always necessary to understand the patient's position in the cultural continuum. Why? This is because the information given may be useful to include the extended discussions on the origin of the disease family, diagnosis and treatment. Consequently, it may be necessary to obtain the consent of the gown up before certain diagnosis and therapy are carried out.

On the other hand, ecological factors usually hasty emergence by placing people in contact with a natural reservoir or host for an infection hitherto unfamiliar but usually already present (often a zootomic or arthropod-borne infection). Thus, by increasing proximity or changing conditions so as to favor an increased population of the microbe orbits natural host. Therefore human activities work to facilitate the spread of diseases. Since the history of infectious diseases has been a history of microbes on the march, often in our wake, and of microbes that have taken advantage of the rich opportunities offered them to thrive, prosper, and spread. Yet the historical processes that have given rise to the emergence of "new" infections throughout history continue today with unabated force; in fact, they are accelerating, because the conditions of modern life ensure that the factors responsible for disease 
emergence are more prevalent than ever before. Speed of travel and global reach are further borne out by studies modeling the spread of influenza epidemics and HIV. It could be said humans are not powerless, however, against this relentless march of microbes.

\section{Technology profile}

The knowledge of technology has put people to different life styles which made them sometimes finding it difficult to readjust. It is said that not everything the glitters is gold. In the same line it is not everything that is put out on the social media that should be admitted. However with a poor know-how the coming of the "android" telephone has put many youngsters to unprepared relations as some spend time swapping from one program to another. A lady recount about her unprepared relationship with a man simply because she wanted an apt modern telephone which gave room to pornography and other wide sentimental programs of an android phone. Given such desires she succumbs as well to the man's option of unprotected sex with uncertainty about his health status, a high risk to vulnerable youthfulness. Through some links some have been connected to people and get into friendships which at times are not positive. Such poor profile of having a phone and not knowledgeable to what programs one need to work with and for what reason is quite impacting among many young people especially the females in Maroua.

\section{Economic profile}

Meanwhile, preparedness to carry out economic activities by some young adolescent as hackers through the town with vegetables, fruits or any commercial items has resulted or get them involve in force sex, rape or sexual attachment to some groups of person considered to be richer. Some cases has given rise to unwanted pregnancy as many of such individuals do not even understand what happened to them since some of these action are carried under the influenced of drugs or are being threaten. Though some of these young ladies even go out with men with the consciousness to make some gain for themselves, some of the men are too old for them and would not want to negotiate any protected sex with them. Besides, one difficulty is that fact that most of the men run about with many females as well. Desperate to come of poverty as some say, they want to raise some money to begin a small business and get rid of them. It may become hard even after they think of quitting. Thus humiliation and poor concession abilities make sexuality a trial among members of the Maroua community.

\section{Academic profile}

With the increasing population as a result of government projects such as the University and other social infrastructures the inflow of people from other parts of the country and outside the country is evident.
Students, teachers, businessmen, administrators and the list continue. Are some of the personalities that come in and consciously or unconsciously influenced the livelihood of many males as well as females. They present several options such as marriage, jobs or some goods or services to these individuals whose mindsets are turned from the different profiles to match that of the former. Such situations put a number into dilemma of either accepting their proposal to get the favour or lose it. In the cloud of all these events some have fallen victims of circumstances. From a student informant this he has to say. With my academic status the young find it difficult to resist given that a way of getting a caliber of my type seems to be a nightmare to many low educated girls in the community.

"We don't have or possess condoms in our hand bags for, if any person knows of that it is not taken lightly. That is if seen by a member of the community of the person proposing, it is view as if you are a commercial sex worker. When we get the opportunity, we cannot arrange condoms at that time as we do not have time for that. We do have sexual intercourse without the use of condo". exclaimed informant. Fatima 23, secondary school student.

In some of our focus groups, informants sensed uncomfortable while speaking about sexual and reproductive health matters. Some could feel a little ease when with friends but with the opposite sex, family, and even with their sexual partners was very difficult. Sometimes men could talk in their small quarter discussions not really as a topic but in their diversity of male matters about women. For the females, they girls found it difficult to start a conversation about it with their sexual companions. Meanwhile the males claimed that it was challenging to keep condoms as they feel ashamed when surrounded by kinfolk or associates.

Meanwhile many unmarried youngsters of both sexes felt afraid and humiliate while purchasing condoms and other contraceptives from indigenous stores, in which case, they are not always available although urban informants appealed that enormous urban stores offer chances for them to buy condom secretly. Some male particpants in our focus groups said that the local salespersons and health service workers know them and their family and are times threaten that their personality could be expose and share evidence about them to close members. Consequently, they live in worried and feel comfortless. Visiting the health Centre is therefore very difficult. Even for those who effectively do and are even informed on preventive when given preventives, they find it shameful to share the story. Besides, our informants made it clear that such humiliation also come from the actions of the medical personnel working in such health clinics or the salespersons who actually put the contraceptive on sale. 
"Sometimes when you ask to buy a condom and you asked what do you want to do with it? It becomes somehow scary and embarrassing. Knowing about condoms and effectively using it, is not an easy one. At times with the salespersons when you often go for the purchase of a condom, they gaze at you otherwise" recount by a female informant. Edith, puff- puff seller, 18 years.

In another report, an informant declare that visiting the hospital for health matter such as headache, fever or an injury as well as any other illness is welcoming. But going for a purchase of a condom hail some negative impression which people could adopt especially if you are a child of a notable or you parents are public figures. In many cases when you buy and go and latter come for an illness that may need diagnosis it is terrifying. Some will start saying things you would wonder and regret going for treatment and sometimes the story of buying preventives could come to the knowledge of your family members or friends during such occurrence.

"During a consultation, the doctor asks many questions which disturbed one's mind especially when you are accompanied by a close member of the family to the hospital or friend. Question such as when was your last date of menses, are you sexually active? Do you use contractive? If yes which? Etc. the fear of answering such questions makes one not to desire to go to the health post of any health complains or one feels an atmosphere of tension and therefore become too shy to open up about our sexual performances with those doctors". Female informant Gladys 20.

\section{Biases Profile}

In many instances of low academic profile be it formal or informal, the population of Maroua and its environs, according to WILPF Cameroon $65^{\text {th }}$ session of February-March "19" p5 in 2012, the Committee on Economic, Social and Cultural Rights had recommended to Cameroon the adoption of a comprehensive anti-discrimination legislation listing all prohibited grounds for discrimination, as set out in article 2, paragraph 2, of the Covenant. Though the report of December 2017 responded to this by submitting the legal framework as regards nondiscrimination relatively developed in Cameroon, discrimination against women is still persistent in custom. WILPF Cameroon $65^{\text {th }}$ session of FebruaryMarch "19" p. 9, indicates that in the issue of Boko Haram crisis and violence in Cameroon, more than 680.000 people have been displaced by the conflict in Cameroon according to OCHA.

Meanwhile, in the Far North, repeated attacks by Boko Haram have created an atmosphere of widespread insecurity. Thus, authorizes report that women no longer want to send their daughters to school or public places like markets for fear of being abducted by Boko Haram since women are particular victims of physical and sexual violence. Or girls are used in suicide attacks. In UNICEF report of April 2017, it is indicated that since January 2014,117 children-with more than $80 \%$ of them girls - have been used in suicide attacks in Nigeria, Nigeria, Niger, Chad and Cameroon. It continued that despite a decline in attacks that are becoming more sporadic and the use of girls as kamikazes also declining in the Far north, marginalized women and girls IDP-seeking to improve their living conditions, are still at risk of being recruited by Boko Haram.

Talking with informants at different participations, it was made known that young people, particularly girls, have deprived intervention and policymaking skills which occasionally lead to insecure sex. Adolescent's females additional recounted that it is not easy to have a conversation about sexual issues with their boyfriends. They indicated that it is less casual of refusing sex if boys ask clearly.

In most small clinics and among health personnel met on the field, their expressions were thoughts to have inadequate combined health-related services whereas other consulting room offers sexual health services at close range. In such cases, the informants were more apprehensive about being known in the clinic by somebody whom they knew already. Situations of identity were quiet embarrassing for total expressing of once feelings about detail connecting health and behaviors. Upon pressing our subject of study, some informants were more comfortable taking because we're seen as strangers and strongly encouraged about confidentially they could speak out their stories. Below are some stories gathered during in-depth interviews with some key persons concerning the biases among individual which is sex related, complexity or social structures. .

When you talk about sex education I heard about this at the age of 24 for the first time when I went to the clinic because I was consulting for some discomfort after a long treatment with traditional herbs without success. I think some of these amenities are carried only in precise places and times. Being there at that particular time is better since those doing the consultation could be government people. In that case it could be free or cheap but if one goes to a public known health centre with some sex-related problems, you should not be surprise to hear a sellout of information. So, just the humiliation of your name going with a sexual illness hinders your relationships with the other girls or opposite sex. Female 16 years, single parent

In another case but more precisely in a group discussion

I have no knowledge of such opportunities but we may feel more relaxed if there are fresh service providers 
especially if there are females. We cannot be comfortable showing parts of our physique to male doctors. I have a cousin who had a problem with her breast and consulted with a male doctor. This man latter because her sex partners. Maybe the shape of her breast attracted him to her and on the other part, he was a civil servant and his money influenced her. And coming back to the younger health service providers as mentioned before, $i$ think there is a complex when you talk with someone closer to your age range than expressing your feelings to your parents' age talk not of sex. It is a very sensitive issue. How will your parents feel about you having sex especially those from strong religious background? (a female student, 23years).

\section{Ecological Profile}

The treatment of certain sickness especially by traditional healers and even traditional rite to prevent girls from desiring sex till they are of marriage age could contribute to tetanus infection since many of these items are crude and are used on many people and is typically traditional without any precaution of sterilization. When kept after the ceremony to be used maybe to another group of persons, it is risky yet no measures are incurred to prevent diseases in the progress. During circumcision, old knives and blades without disaffections are used to circumcise a number of children and in the process disinfection measures are not put in place given that they are old or use on many children at a time.

Talking on the rite of initiation of young boys and girls to choose their marriage partner which is highly respected in the Extreme North region of Cameroon, it could be considered as another route health risk that encourage propagation of diseases. By this many youngsters are given to sex even before marriage and when married if not satisfied with one another they separate and remarry to another person. What if one of the partners is affected? he/she would infect others since in their separation and remarry process no test are carried out. Such whimsical sex behavior in a situation of disease, individuals would only help in the spread of the infection. More and more divorce is so rampant in the Extreme North such that a market is even named after this phenomenon in the region. Marche de femme. This market is place where women go and sell their household utensils after divorcing with their husbands given that she come in to marriage with them. Selling her utensils gives room for another marriage and partner which goes on even without checks.

\section{Impact of Low profiles on the female sexual active Youngesters}

According to World Health Organisation, sexual health is defined as a state of physical, emotional, mental and social wellbeing in relation to sexuality; it is not just the absence of disease, dysfunction or infirmity. Sexual health therefore requires a positive and respectful approach to sexuality and sexual relationships, as well as the possibility of having pleasurable and safe sexual experiences, free of coercion, discrimination and violence. For sexual health to be attained and maintained, the sexual rights of all persons must be respected, protected and fulfilled.

However, most adults are sexually active and good sexual health matters to individuals and communities. To that effect, it should be indicated here that sexual health needs differ according to factors such as age, gender, sexuality and ethnicity. Yet, there are certain essential condition needs shared by everyone comprising high quality information and education. Hence such is enabling people to make knowledgeable accountable choices, and access to high quality services, management and involvements. Nevertheless, taking a look at our study area in particular, and Cameroon as a whole sexual health, reproductive health and human immunodeficiency virus (HIV) embraces the delivery of guidance and facilities about contraception, sexually transmitted infections (STIs), HIV and termination of pregnancy. To that effect the consequences involving low profile in sexually include: unintended pregnancies, abortions. Meanwhile, Psychological consequences of sexual coercion and abuse, poor educational, social and economic opportunities for teenage mothers, young fathers and their children, HIV amongst others are not left out. In confirmation to the above scenario, Findings from the national surveys of sexual attitudes and lifestyles (Natsal) show most young people become sexually active and start forming relationships between the ages of 16 and 24. In this light, young people in these age groups have significantly higher rates of poor sexual health, including STIs and abortions than older people.

Meanwhile in 2015, HIV in the United Kingdom 2016 report estimated 101,200 people were living with HIV in the United Kingdom of which it is projected that $13 \%$ are unaware of their infection and remain at risk of infecting others and are unable to benefit from effective treatment. Moreso, it has long been recognised that gonorrhea is at risk of becoming an untreatable disease due to the ongoing threat of antimicrobial resistance. Being one of the neglected tropical diseases, many people in the extreme North like in many parts of Cameroon when affected get in to clandestine treatment and in most cases; serious measures for effect treatment are not put in place. What even make things worse is the fact that many always attribute its causes to superstition and concentrate of carry out rituals than handling the disease on time.

\section{DiscuSsiOnS}

Given the empirical status of the high number of low profile persons in our research area, the 
challenge is quite visible and need necessary intervention through media, community workers, cultural platform, information and a wide range of communication with a determinant change of mindset. Therefore, for a good realization of such a vital effort to be put in place, the individual, groups, learn, uneducated, songs, and all need to be implicated. Thus the following could take different measures to pass the message.

\section{Core principles for healthcare professionals \\ Healthcare professionals should:}

- distinguish the requests of individuals, communities and populations connected to sexual health, reproductive health and HIV

- reflect on the means and the amenities accessible in the health and welfare scheme in order to encourage good sexual and procreative health

- comprehend precise undertakings or interferences which can avoid poor sexual health, reproductive health and HIV

Notwithstanding the above Interventions, at the population level

Healthcare experts would need to be conscious of the involvements at population level, which include:

- construction of an authentic and exposed philosophy where one and all is able to make knowledgeable and answerable sets about dealings and sex to lessen the humiliation linked with sexual conditions and HIV

In education, it would be necessary safeguarding children to obtain good quality sex and affiliations education at home-based, school, social gatherings and in the unrestricted sessions. In addition, statutory assistance and extra advice produced by the Department for Education could be explore and use to guide children in their daily routine in relation to sex.

- Creating cognizance that suggest contraception and STI and HIV behavior are delivered free from preparation care to decrease the risk of undesirable pregnancy and headlong spread of infection

- guaranteeing that avoidance is highlighted and people are driven to exercise safe sex, as well as using contraception and condoms

- certifying analysis for HIV and STIs is efficiently endorsed in at-risk inhabitants

\section{Community level}

Community health professionals and providers of specialist services can have an impact by:

- ensuring local authorities commission services for the full range of contraception, the testing and treatment of STIs and provision of condoms for the benefit of everyone in the community

- protecting patients from reinfection with partner notification - an essential component of STI management and control
- offering chlamydia testing to young people as a routine part of every primary care and sexual health consultation; the goal of the national chlamydia screening programme (NCSP)

- ensuring easy access to sexual health advice, free condoms, and testing for HIV and other STIs for young people and other high risk groups in a range of accessible settings with condom distribution schemes

\section{Family and individual level}

Healthcare professionals can have an impact on an individual level by:

- providing information about the full range of contraceptive methods and promoting prompt access to the method that best suits their needs; see the Sexist website for downloadable information leaflets

- ensuring that women seeking an abortion have easy, quick and confidential access to services

- ensuring people understand the different STIs, associated potential consequences and how to reduce the risk of transmission; the Sexist website provides a number of useful resources

- $\quad$ providing information about where to get prompt access to HIV testing

- ensuring people who are diagnosed with HIV receive prompt referral into care and high quality treatment services

\section{Way forward}

Besides the need for more research on sexuality and the cultural notion of reproductive health especially in the Extreme North of Cameroon to be examined it is also necessary to look at the role of peers in connection to making decisions on alcohol, drugs, sex, and romantic relationship. On the field we noticed that a good number of young individuals who were informants use smoking and drinking habits to impact their folks. Contributors equally indicated that peer's groups were quite instrumental in the knowledge of sex as they find it humiliation or suspicious when they ask from older persons. Based on the idea that some generalized information they get from one another, many falls as victims to many problems of sexuality since they do not understand that each individual has a unique reproductive which cannot be the same with the other peer. Poor academic profile hinder persons to know that even among persons with similar behaviours, their reproductive cycles varies, giving way to different challenges in sexuality.

Considerations for organize workshops to train grassroots women to conduct participatory research should be highlighted as this would enable them to cultivate talents to disapprovingly examine their prevailing circumstances. As a result it would motivate them to carry out collective action. That is to say the sexualized gaze will appears through a different but still 
interesting mechanism. Thus participatory research is view as vital as oral and personal experiences in most cases path the way for a future research agenda with a traditional blend, other researchers will be able cross check achievement or losses with passing time. The same helps as a roadmap for the informants to see and share the results with them in a language and manner that can be acknowledge so that their mindset as well could be orientated. Participatory research as an approach would consequently necessitate:

\section{Spreading evidence;}

- creating and circulating material flyers concerning females' rights;

- mentioning to women in all national and UN statistics;

- gathering oral past of women;

- write down and examining successful and unsuccessful programmes of the women's movements as well as their own contribution which is quite instrumental for dynamics for policy makers.

\section{Conclusion}

Educational Schemes for the prospect consensus are preferably seen as a continuous holistic process with rational, psychosomatic, monetary and governmental scopes in order to attain freedom since women are the most highly affected in this domain. In view with the intricacy of the political, communal and global networking, it would be imperative to thoroughly deliberate about the approaches and tangible suggestions for impending achievement if one anticipations to attain such aim. Thus a complexity policy on training, exploration/documents, crusades, interacting, persuading strategies, drill and broadcasting need to be emphasis. Education being the core, the formal and non-formal education systems would need to be considered. Meanwhile, it would be of great significant to evaluate the gender gratified and to determine the way in which it is spoken in the cultural and educational system.

\section{REFERENCES}

1. International Communications Research. (2007). Teen attitudes toward sex. Survey from the National Campaign to Prevent Teen Pregnancy.

Retrieved May 31, 2007 from

http://www.teenpregnancy.org/.

2. Jecker, N. S., Carrese, J. A., \& Pearlman, R. A. (1995). Caring for patients in cross-cultural settings. Hastings Center Report, 25(1), 6-14.

3. Kaestle, C.E. (2006). Sexual health of young adults and age at first intercourse. Unpublished doctoral dissertation, University of North Carolina at Chapel Hill.

4. Lester, C., \& Allan, A. (2006). Teenage sexual health needs: Asking the consumers. Health Education, 106, 315-326.

5. Lynch L. J. (2002). More HIV/AIDS education needed in schools. Chicago Weekend, 32, 1.

6. Miller, K.E. (2007). Public opinion vs. science concerning sex education. American Family Physician, 75, 1609.

7. Pokharel, S., Kulczycki, A., \& Shakya, S. (2006). School-based sex education in Western Nepal: Uncomfortable for both teachers and students. Reproductive Health Matters, 14, 156-162.

8. Pradhan, Ajit and Molly (2003). Adolescent Reproductive Health in Nepal: Status, Policies, Programs, and Issues Imprint POLICY Project, January 2003, 27 pp. Url .http://www.policyproject.com/pubs/countryreports /ARH_Nepal.pdf

9. UNFPA (2017) in Crisis to reproductive health often determines if women and girls live or die says, UNFPA Executive Director. Retrieved from https:/ Nigeria. UNFPA/org/en/news crisis-accessreproductive- health- care- often- determines- if women- or -girls- live- or- die- says.

10. UNHCR (2017) Nigeria: sex and gender based violence (SGBV) monthly update-April . Retrieved from https://. relief web.int/report-nigeria/nigeriasexual- gender- based -violence -sgbv- monthly update- april- 2017.

11. UNICEF Child (2017) Child Labour. retrieved from https:/data.UNICEF.org/topic/childprotection/child-

12. United Nations Population Fund, 1998. UNFPA gender theme group, Geneva: United Nations Population Fund.

13. Women's International League for Peace and Freedom - Cameroon (2019) Women's Economic, Social and Cultural Rights in Cameroon. $65^{\text {th }}$ session parallel report to the UN Committee on Economic and Cultural Rights. February. 